\title{
A NOTE ON ENTIRE PSEUDO-HOLOMORPHIC CURVES AND THE PROOF OF CARTAN-NOCHKA'S THEOREM
}

\author{
JUNJIRO NOGUCHI
}

To the memory of Professor Nobuyuki Suita

\section{Introduction}

The purpose of this note is twofold. The first is to prove a lemma on differentials for entire pseudo-holomorphic curves in a compact almost complex manifold (see Lemma 2.1), which is an analogue to Nevanlinna's lemma on logarithmic derivative and also to a lemma on holomorphic 1-forms which had been conjectured by A. Bloch [B126] and was proved by T. Ochiai [Oc77].

The second is to give a complete proof of Cartan-Nochka's Theorem with truncated counting functions and with small error term " $S_{f}(r)=O\left(\log ^{+} r+\right.$ $\left.\log ^{+} T_{f}(r)\right) \|$ " by a simple Cartan method (see Theorem 3.1). This was what Nochka [Nc83] stated the theorem with a sketch of the proof. So far there has been no literature of the complete full proof which is accessible to wider audience, while there are, I learned orally, a longer paper of Nochka in Russian other than [Nc82a], [Nc82b] and [Nc83], and a proof based on the same idea as the present one in the book $[\mathrm{Ng} 03]$ in Japanese. We will show the theorem in a form slightly more general than those in the above mentioned references.

We also give some generalization to the case where the domain is an analytic ramified covering space over $\mathbf{C}^{m}$ (see Theorem 3.18).

Acknowledgement. Because of the importance of Cartan-Nochka's Theorem Professor Y. Aihara suggested the author to write up the present note that should be useful. The author is grateful for him and his suggestion.

\section{Entire pseudo-holomorphic curves}

The problem of Kobayashi hyperbolicity for pseudo-holomorphic curves are intensively studied by several authors (cf., e.g., R. Debalme and S. Ivashkovich [DI01] S. Kobayashi [Ko01], [Ko03], [Ko04]). The analogue of Brody's Theorem holds for pseudo-holomorphic curves (cf. [Ko03], [Ko04]). Henceforth the Kobayashi hyperbolicity of a compact almost complex manifold $M$ is in-

Received April 23, 2004; revised July 23, 2004. 
ferred from the non-existence of a non-constant entire pseudo-holomorphic curve $f: \mathbf{C} \rightarrow M$.

In the complex analytic case, Nevanlinna theory seems to be the best approach for the non-existence problem of $f$ (cf. S. Kobayashi [Ko98] Introduction), where the so-called Nevanlinna's lemma on logarithmic derivative plays an essential role and provides a counter part to Schwarz' Lemma in the theory of Kobayashi hyperbolicity. In the proof of Bloch-Ochiai's Theorem (cf. [NO $\left.\frac{84}{90}\right]$ ) a lemma on holomorphic differentials, which had been conjectured by [B126] and was proved by [Oc77], was important and played an exactly similar role to Nevanlinna's lemma on logarithmic derivative. The purpose of this section is to show such a lemma for entire pseudo-holomorphic curves (see Lemma 2.1).

Let $M$ be a compact almost complex manifold and let $h=\sum h_{v \bar{\mu}} d x^{v} d \bar{x}^{\mu}$ be a fixed hermitian metric on $M$ with the associated $(1,1)$-form $\omega=\sum h_{v \bar{\mu}} \frac{i}{2} d x^{v} \wedge$ $d \bar{x}^{\mu}$. Let $f: \mathbf{C} \rightarrow M$ be an entire pseudo-holomorphic curve. We define the order function of $f$ with respect to $h$ by

$$
T_{f}(r ; h)=\int_{0}^{r} \frac{d t}{t} \int_{|z|<t} f^{*} \omega .
$$

For a smooth differential 1-form $\eta$ on $M$ we have the decomposition $\eta=\eta^{\prime}+\eta^{\prime \prime}$ to the $(1,0)$-form $\eta^{\prime}$ and $(0,1)$-form $\eta^{\prime \prime}$. We set

$$
\begin{gathered}
f^{*} \eta=\eta_{f}^{\prime} d z+\eta_{f}^{\prime \prime} d \bar{z} \\
m_{f}(r ; \eta)=\int_{|z|=r} \log ^{+} \sqrt{\left|\eta_{f}^{\prime}\right|^{2}+\left|\eta_{f}^{\prime \prime}\right|^{2}} \frac{d \theta}{2 \pi} .
\end{gathered}
$$

Here $\log ^{+} s=\max \{\log s, 0\}$ for $s \in \mathbf{R}^{+}=\{s \in \mathbf{R} ; s \geqq 0\}$.

Lemma 2.1. Let $f: \mathbf{C} \rightarrow M$ be a pseudo-holomorphic curve and let $\eta$ be a smooth differential 1-form on $M$. Then we have

$$
m_{f}(r ; \eta) \leqq \delta \log r+2 \log ^{+} T_{f}(r ; h) \|_{\delta} \quad(0<\delta<1),
$$

where the symbol " $\|_{\delta}$ " stands for the stated inequality to hold for all $r>0$ outside a Borel subset dependent on $\delta>0$ with finite Lebesgue measure.

We need the following, called Borel's Lemma (cf., e.g., [NO $\left.\frac{84}{90}\right]$ ).

LEMMA 2.2. Let $\phi(r)$ be a continuous, increasing function on $\mathbf{R}^{+}$such that $\phi\left(r_{0}\right)>0$ for some $r_{0} \in \mathbf{R}^{+}$. Then for an arbitrary small $\delta>0$ we have

$$
\frac{d}{d r} \phi(r)<\phi(r)^{1+\delta} \|_{\delta}
$$

To prove Lemma 2.1 we may assume that $\eta$ is a $(1,0)$-form. We set 


$$
f^{*} \eta=\eta_{f}(z) d z, \quad f^{*} \omega=s(z) \frac{i}{2} d z \wedge d \bar{z}, \quad z \in \mathbf{C} .
$$

Since the length $\|\eta\|_{h}$ of $\eta$ with respect to $h$ is bounded, there is a constant $C>0$ such that

$$
\left|\eta_{f}(z)\right|^{2} \leqq 2 \pi C s(z)
$$

Let $0<\delta<1$. Using the concavity of the logarithmic function and Lemma 2.2, we have

$$
\begin{aligned}
m_{f}(r ; \eta) & =\int_{|z|=r} \log ^{+}\left|\eta_{f}\right| \frac{d \theta}{2 \pi} \leqq \frac{1}{2} \int_{|z|=r} \log \left(1+\left|\eta_{f}\right|^{2}\right) \frac{d \theta}{2 \pi} \\
& \leqq \frac{1}{2} \log \left(1+\int_{|z|=r}\left|\eta_{f}\right|^{2} \frac{d \theta}{2 \pi}\right) \leqq \frac{1}{2} \log \left(1+C \int_{|z|=r} s(z) d \theta\right) \\
& \leqq \frac{1}{2} \log \left(1+\frac{C}{r} \frac{d}{d r} \int_{\Delta(r)} s(z) t d t \wedge d \theta\right) \\
& \leqq \frac{1}{2} \log \left(1+\frac{C}{r}\left(\int_{\Delta(r)} s(z) \frac{i}{2} d z \wedge d \bar{z}\right)^{1+\delta}\right) \|_{\delta} \\
& \leqq \frac{1}{2} \log \left(1+C r^{\delta}\left(\frac{d}{d r} \int_{1}^{r} \frac{d t}{t} \int_{\Delta(t)} f^{*} \omega\right)^{1+\delta}\right) \|_{\delta} \\
& \leqq \frac{1}{2} \log \left(1+C r^{\delta}\left(\int_{1}^{r} \frac{d t}{t} \int_{\Delta(t)} f^{*} \omega\right)^{(1+\delta)^{2}}\right) \|_{\delta} \\
& \leqq \frac{1}{2} \log \left(1+C r^{\delta}\left(T_{f}(r ; h)\right)^{(1+\delta)^{2}}\right) \|_{\delta} \\
& \leqq \delta \log r+2 \log ^{+} T_{f}(r ; h) \|_{\delta} .
\end{aligned}
$$

Remark. It is interesting to observe that the complex analyticity of $\eta$ is completely irrelevant to the above Lemma 2.1. As in Nevanlinna theory in complex analysis Lemma 2.1 is expected to apply to the Kobayashi hyperbolicity problem.

\section{Cartan-Nochka's Theorem}

Let $H_{j}, 1 \leqq j \leqq q$ be hyperplanes of $\mathbf{P}^{n}(\mathbf{C})$ defined by

$$
H_{j}: \quad \sum_{k=0}^{n} h_{j k} w^{k}=0, \quad 1 \leqq j \leqq q,
$$


where $\left[w^{0}, \ldots, w^{n}\right]$ is a homogeneous coordinate system of $\mathbf{P}^{n}(\mathbf{C})$. Set the index set $Q=\{1, \ldots, q\}$. For a subset $R \subset Q,|R|$ denotes its cardinality.

Definition. Let $N \geqq n$ and $q \geqq N+1$. We say that $H_{j}, j \in Q$ are in $N$ subgeneral position if for every subset $R \subset Q$ with $|R|=N+1$

$$
\bigcap_{j \in R} H_{j}=\emptyset \text {. }
$$

If they are in $n$-subgeneral position, we simply say that they are in general position.

Being in $N$-subgeneral position is equivalent to that for an arbitrary $(N+1, n+1)$-matrix $\left(h_{j k}\right)_{j \in R, 0 \leqq k \leqq n}$

$$
\operatorname{rank}\left(h_{j k}\right)_{j \in R, 0 \leqq k \leqq n}=n+1 .
$$

Let $f: \mathbf{C}^{m} \rightarrow \mathbf{P}^{n}(\mathbf{C})$ be a meromorphic mapping. Let $z=\left(z_{j}\right)$ be the natural coordinate system of $\mathbf{C}^{m},\|z\|=\sqrt{\sum_{j}\left|z_{j}\right|^{2}}$ and let $\omega$ be the Fubini-Study metric form on $\mathbf{P}^{n}(\mathbf{C})$. We define the order function $T_{f}(r)$ with respect to $\omega$ by

$$
T_{f}(r)=\int_{0}^{r} \frac{d t}{t^{2 m-1}} \int_{\|z\|<t}\left(\frac{i}{2 \pi} \partial \bar{\partial}\|z\|^{2}\right)^{2 m-2} \wedge f^{*} \omega .
$$

Cf. $\left[\mathrm{NO}_{90}^{84}\right]$ for general notation in Nevanlinna theory. We denote a such small term by $S_{f}(r)$ that for an arbitrarily small positive number $\delta$

$$
S_{f}(r) \leqq \delta \log r+O\left(\log T_{f}(r)\right) \|_{\delta} .
$$

For a hyperplane $H \subset \mathbf{P}^{n}(\mathbf{C})$ such that $H \not \supset f\left(\mathbf{C}^{m}\right)$ we have the pull-backed divisor $f^{*} H$ on $\mathbf{C}^{m}$ and the irreducible decomposition $f^{*} H=\sum_{j} v_{j} Z_{j}$. We define the truncated divisor $\left(f^{*} H\right)_{[k]}$ to the level $k \in \mathbf{N} \cup\{\infty\}$ by

$$
\left(f^{*} H\right)_{[k]}=\sum_{j} \min \left\{v_{j}, k\right\} Z_{j} .
$$

We define the counting function $N_{k}\left(r, f^{*} H\right)$ of the divisor $\left(f^{*} H\right)_{[k]}$ by

$$
N_{k}\left(r, f^{*} H\right)=\int_{1}^{r} \frac{d t}{t^{2 m-1}} \int_{\left(f^{*} H\right)_{[k]} \cap\{\|z\|<t\}}\left(\frac{i}{2 \pi} \partial \bar{\partial}\|z\|^{2}\right)^{2 m-2} .
$$

and set $N\left(r, f^{*} H\right)=N_{\infty}\left(r, f^{*} H\right)$ (cf. [NO $\left.\left.\frac{84}{90}\right],[\mathrm{Fu} 93]\right)$.

TheOREM 3.1 ([Nc83] for $m=1$ ). Let $f: \mathbf{C}^{m} \rightarrow \mathbf{P}^{n}(\mathbf{C})$ be a linearly nondegenerate meromorphic mapping. Let $H_{j}, 1 \leqq j \leqq q$ be hyperplanes of $\mathbf{P}^{n}(\mathbf{C})$ in $N$-subgeneral position. Then we have

$$
(q-2 N+n-1) T_{f}(r) \leqq \sum_{j=1}^{q} N_{n}\left(r, f^{*} H_{j}\right)+S_{f}(r) .
$$


Remark. i) $(m=1)$ The case of $m=1$ is essential. H. Cartan [Ca33] proved this when $H_{j}, 1 \leqq j \leqq q$ are in general position.

ii) $(m \geqq 1)$ By Weyl-Ahlfors' method Chen [Ch90] proved

$$
(q-2 N+n-1) T_{f}(r, L)+\frac{N+1}{n+1} N(r,(W(f))) \leqq \sum_{j=1}^{q} N\left(r, f^{*} H_{j}\right)+S_{f}(r),
$$

where $(W(f))$ denotes the divisor defined by the Wronskian of $f$ (see (3.6)). After this formulation it is unable to deduce (3.2).

iii) $(m=1)$ By Weyl-Ahlfors' method combined with his own technique $\mathrm{H}$. Fujimoto $[\mathrm{Fu} 93]$ proved that for an arbitrary $\varepsilon>0$

$$
(q-2 N+n-1) T_{f}(r) \leqq \sum_{j=1}^{q} N_{n}\left(r, f^{*} H_{j}\right)+\varepsilon T_{f}(r) \|_{\varepsilon} .
$$

Here, the estimate of the small error term is not as good as in (3.2); it is noticed that the type of error term is in general deeply related to the possible truncation level of counting functions in the right-hand side of (3.2) (see [NWY02] Example (5.36)).

Let $H_{j}, j \in Q$ be hyperplanes of $\mathbf{P}^{n}(\mathbf{C})$ in $N$-subgeneral position. For $R \subset Q$ we set

$$
\begin{aligned}
V(R) & =\text { the vector subspace spanned by }\left(h_{j k}\right)_{0 \leqq k \leqq n}, j \in R \text { in } \mathbf{C}^{n+1}, \\
\operatorname{rk}(R) & =\operatorname{dim} V(R), \quad \operatorname{rk}(\emptyset)=0 .
\end{aligned}
$$

We recall now lemmas due to Nochka (see [Nc83], [Ch90], [Fu93]).

Lemma 3.3 ([Nc83], [Ch90], [Fu93]). Let $H_{j}, j \in Q$ be hyperplanes of $\mathbf{P}^{n}(\mathbf{C})$ in $N$-subgeneral position, and assume that $q>2 N-n+1$. Then there are positive rational constants $\omega(j), j \in Q$ satisfying the following:

(i) $0<\omega(j) \leqq 1, \forall j \in Q$.

(ii) Setting $\tilde{\omega}=\max _{j \in Q} \omega(j)$, one gets

$$
\sum_{j=1}^{q} \omega(j)=\tilde{\omega}(q-2 N+n-1)+n+1 .
$$

(iii) $\frac{n+1}{2 N-n+1} \leqq \tilde{\omega} \leqq \frac{n}{N} \cdot 1$

(iv) For $R \subset Q$ with $0<|R| \leqq N+1, \sum_{j \in R} \omega(j) \leqq \operatorname{rk}(R)$.

\footnotetext{
${ }^{1}$ The bound $\frac{n}{N}$ which is better than the original one $\frac{n+1}{N+1}$, was suggested by N. Toda by a careful check of the proof.
} 
The above $\omega(j)$ are called the Nochka weights, and $\tilde{\omega}$ the Nochka constant.

Lemma 3.4 ([Nc83], [Ch90], [Fu93]). Let $q>2 N-n+1$, and let $\left\{H_{j}\right\}_{j \in Q}$ be a family of hyperplanes of $\mathbf{P}^{n}(\mathbf{C})$ in $N$-subgeneral position. Let $\{\omega(j)\}_{j \in Q}$ be its Nochka weights.

Let $E_{j} \geqq 1, j \in Q$ be arbitrarily given numbers. Then for every subset $R \subset Q$ with $0<|R| \leqq N+1$, there are distinct indices $j_{1}, \ldots, j_{\mathrm{rk}(R)} \in R$ such that $\operatorname{rk}\left(\left\{j_{l}\right\}_{l=1}^{\operatorname{rk}(R)}\right)=\operatorname{rk}(R)$ and

$$
\prod_{j \in R} E_{j}^{\omega(j)} \leqq \prod_{l=1}^{\mathrm{rk}(R)} E_{j_{l}}
$$

Let $f: \mathbf{C}^{m} \rightarrow \mathbf{P}^{n}(\mathbf{C})$ be a linearly nondegenerate meromorphic mapping. Fix a homogeneous coordinate system $w=\left[w^{0}, \ldots, w^{n}\right]$ of $\mathbf{P}^{n}(\mathbf{C})$ and let $f(z)=$ $\left[f^{0}(z), \ldots, f^{n}(z)\right]$ be a reduced representation.

Assume that $H_{j}$ are defined by

$$
\begin{aligned}
H_{j}: \quad \hat{H}_{j}(w) & =\sum_{k=0}^{n} h_{j k} w^{k}=0, \quad 1 \leqq j \leqq q, \\
\left\|\hat{H}_{j}\right\| & =\left(\sum_{k}\left|h_{j k}\right|^{2}\right)^{1 / 2}=1, \quad \frac{\left|\hat{H}_{j}(w)\right|}{\|w\|} \leqq 1 .
\end{aligned}
$$

After [Fu85] and [Ng97] §2 (b), we define the Wronskian $W(f)=$ $W\left(f^{0}, \ldots, f^{n}\right) \not \equiv 0$, and the logarithmic Wronskian $\Delta\left(f^{0}, \ldots, f^{n}\right)$ as follows:

$$
\begin{gathered}
W\left(f^{0}, \ldots, f^{n}\right)=\left|\begin{array}{ccc}
f^{0} & \cdots & f^{n} \\
D^{(1)} f^{0} & \cdots & D^{(1)} f^{n} \\
\vdots & \vdots & \vdots \\
D^{(n)} f^{0} & \cdots & D^{(n)} f^{n}
\end{array}\right|, \\
\Delta\left(f^{0}, \ldots, f^{n}\right)=\left|\begin{array}{ccc}
1 & \cdots & 1 \\
\frac{D^{(1)} f^{0}}{f^{0}} & \cdots & \frac{D^{(1)} f^{n}}{f^{n}} \\
\vdots & \vdots & \vdots \\
\frac{D^{(n)} f^{0}}{f^{0}} & \cdots & \frac{D^{(n)} f^{n}}{f^{n}}
\end{array}\right| .
\end{gathered}
$$

Here $D^{(j)}=\left(\frac{\partial}{\partial z^{1}}\right)^{\alpha_{1}(j)} \cdots\left(\frac{\partial}{\partial z^{m}}\right)^{\alpha_{m}(j)}$ are some partial differentiations of order at most $j$. Because of the choice of $D^{(j)}$ we have the following functional equations for a meromorphic function $g$ on $\mathbf{C}^{m}$ and $A \in \mathrm{GL}(n+1, \mathbf{C})$ : 


$$
\begin{aligned}
W\left(g f^{0}, \ldots, g f^{n}\right) & =g^{n+1} W\left(f^{0}, \ldots, f^{n}\right), \\
W\left(\left(f^{0}, \ldots, f^{n}\right) A\right) & =W\left(f^{0}, \ldots, f^{n}\right) \times(\operatorname{det} A), \\
\Delta\left(g f^{0}, \ldots, g f^{n}\right) & =\Delta\left(f^{0}, \ldots, f^{n}\right), \\
\Delta\left(1, \frac{f^{1}}{f^{0}}, \ldots, \frac{f^{n}}{f^{0}}\right) & =\Delta\left(f^{0}, \ldots, f^{n}\right) .
\end{aligned}
$$

The following lemma is a key to get the correct truncation level of counting functions:

Lemma 3.8 ([Fu93] Lemma 3.2.13). Let the notation be as above. Then the following inequality holds as divisors on $\mathbf{C}^{m}$ with rational coefficients:

$$
\sum_{j \in Q} \omega(j)\left(\hat{H}_{j} \circ f\right)-\left(W\left(f^{0}, \ldots, f^{n}\right)\right) \leqq \sum_{j \in Q} \omega(j)\left(f^{*} H_{j}\right)_{[n]} .
$$

Remark. H. Fujimoto [Fu93] gave a detailed proof of this lemma for $m=1$, and the same proof works for general $m \geqq 1$.

For a subset $R \subset Q,|R|=n+1$ we define $W\left(\left(\hat{H}_{j} \circ f, j \in R\right)\right)$ and $\Delta\left(\left(\hat{H}_{j} \circ f\right.\right.$, $j \in R)$ ) as Wronskian and logarithmic Wronskian of $\hat{H}_{j} \circ f, j \in R$, respectively.

Now we prove a key lemma of the proof of Theorem 3.1:

Lemma 3.9. Let $q>2 N-n+1$ and let $\omega(j), \tilde{\omega}$ be the Nochka weights and constant of $\left\{H_{j}\right\}_{j \in Q}$, respectively. Then there is a positive constant $C$ dependent on $\left\{\hat{H}_{j}\right\}_{j \in Q}$ such that for an arbitrary $z \in \mathbf{C}^{m} \backslash\left\{\prod_{j \in Q} \hat{H}_{j} \circ f=0\right\}$

$$
\begin{aligned}
\|f(z)\|^{\tilde{\omega}(q-2 N+n-1)} \leqq & C \frac{\prod_{j \in Q}\left|\hat{H}_{j}(f(z))\right|^{\omega(j)}}{\left|W\left(f^{0}(z), \ldots, f^{n}(z)\right)\right|} \\
& \times\left\{\sum_{R \subset Q,|R|=n+1}\left|\Delta\left(\left(\hat{H}_{j} \circ f(z), j \in R\right)\right)\right|\right\} .
\end{aligned}
$$

Proof. By the definition of $N$-subgeneral position, for an arbitrary point $w \in \mathbf{P}^{n}(\mathbf{C})$, there exists $S \subset Q,|S|=q-N-1$ such that $\prod_{j \in S} \hat{H}_{j}(w) \neq 0$. Therefore, there is a constant $C_{1}>0$ such that

$$
C_{1}^{-1}<\sum_{|S|=q-N-1} \prod_{j \in S}\left(\frac{\left|\hat{H}_{j}(w)\right|}{\|w\|}\right)^{\omega(j)}<C_{1}, \quad \forall w \in \mathbf{P}^{n}(\mathbf{C}) .
$$

We consider those $w \in \mathbf{P}^{n}(\mathbf{C})$ such that $\prod_{j \in Q} \hat{H}_{j}(w) \neq 0$. Setting $R=Q \backslash S$, we have

$$
\prod_{j \in S}\left(\frac{\left|\hat{H}_{j}(w)\right|}{\|w\|}\right)^{\omega(j)}=\prod_{j \in R}\left(\frac{\|w\|}{\left|\hat{H}_{j}(w)\right|}\right)^{\omega(j)} \cdot \frac{\prod_{j \in Q}\left|\hat{H}_{j}(w)\right|^{\omega(j)}}{\|w\|^{\sum_{j \in Q}(j)}} .
$$


By making use of Lemma 3.3 (ii) and $\operatorname{rk}(R)=n+1$ for $R$, we obtain a subset $\left\{j_{1}, \ldots, j_{n+1}\right\}=R^{\circ} \subset R$ given by Lemma 3.4 , so that

$$
\begin{aligned}
\prod_{j \in S}\left(\frac{\left|\hat{H}_{j}(w)\right|}{\|w\|}\right)^{\omega(j)} & \leqq\left(\prod_{j \in R^{\circ}} \frac{\|w\|}{\left|\hat{H}_{j}(w)\right|}\right) \cdot \frac{\prod_{j \in Q}\left|\hat{H}_{j}(w)\right|^{\omega(j)}}{\|w\|^{\tilde{\omega}(q-2 N+n-1)+n+1}} \\
& =\frac{1}{\prod_{j \in R^{\circ}}\left|\hat{H}_{j}(w)\right|} \cdot \frac{\prod_{j \in Q}\left|\hat{H}_{j}(w)\right|^{\omega(j)}}{\|w\|^{\tilde{\omega}(q-2 N+n-1)}} .
\end{aligned}
$$

Because of Wronskian's property (3.7), there is a constant $c\left(R^{\circ}\right)>0$ such that

$$
c\left(R^{\circ}\right) \frac{\left|W\left(\left(\hat{H}_{j} \circ f, j \in R^{\circ}\right)\right)\right|}{\left|W\left(f^{0}, \ldots, f^{n}\right)\right|}=1 .
$$

For $z \in \mathbf{C}^{m} \backslash\left\{\prod_{j \in Q} \hat{H}_{j} \circ f=0\right\}$ this with (3.11) implies

$$
\begin{aligned}
\prod_{j \in S}\left(\frac{\left|\hat{H}_{j} \circ f(z)\right|}{\|f(z)\|}\right)^{\omega(j)} \leqq & c\left(R^{\circ}\right) \frac{1}{\|f(z)\|^{\tilde{\omega}(q-2 N+n-1)}} \\
& \cdot \frac{\prod_{j \in Q}\left|\hat{H}_{j} \circ f(z)\right|^{\omega(j)}}{\left|W\left(f^{0}(z), \ldots, f^{n}(z)\right)\right|} \cdot \frac{\left|W\left(\left(\hat{H}_{j} \circ f(z), j \in R^{\circ}\right)\right)\right|}{\prod_{j \in R^{\circ}}\left|\hat{H}_{j} \circ f(z)\right|} \\
= & c\left(R^{\circ}\right) \frac{1}{\|f(z)\|^{\tilde{\omega}(q-2 N+n-1)}} \\
& \cdot \frac{\prod_{j \in Q}\left|\hat{H}_{j} \circ f(z)\right|^{\omega(j)}}{\left|W\left(f^{0}(z), \ldots, f^{n}(z)\right)\right|} \cdot\left|\Delta\left(\left(\hat{H}_{j} \circ f(z), j \in R^{\circ}\right)\right)\right| .
\end{aligned}
$$

Hence, setting $C=C_{1} \max _{R^{\circ}}\left\{c\left(R^{\circ}\right)\right\}$, we obtain the desired inequality. Q.E.D.

Proof of Theorem 3.1. We may assume that $q-2 N+n-1>0$. By Lemmas 3.9, 3.8, and Jensen's formula we have

$$
\begin{aligned}
\tilde{\omega}(q- & 2 N+n-1) T_{f}(r) \\
\leqq & \sum_{j=1}^{q} \omega(j) N_{n}\left(r, f^{*} H_{j}\right) \\
& +\frac{1}{2 \pi} \int_{|z|=r} \log \left(\sum_{R \subset Q,|R|=n+1}\left|\Delta\left(\left(\hat{H}_{j} \circ f(z), j \in R\right)\right)\right|\right) d \theta+O(1)
\end{aligned}
$$




$$
\begin{aligned}
\leqq & \tilde{\omega} \sum_{j=1}^{q} N_{n}\left(r, f^{*} H_{j}\right) \\
& +\frac{1}{2 \pi} \int_{|z|=r} \log \left(\sum_{R \subset Q,|R|=n+1}\left|\Delta\left(\left(\hat{H}_{j} \circ f(z), j \in R\right)\right)\right|\right) d \theta+O(1) .
\end{aligned}
$$

It follows that

$$
\begin{aligned}
& (q-2 N+n-1) T_{f}(r) \\
& \leqq \\
& \quad \sum_{j=1}^{q} N_{n}\left(r, f^{*} H_{j}\right) \\
& \quad+\frac{1}{2 \pi \tilde{\omega}} \int_{|z|=r} \log \left(\sum_{R \subset Q,|R|=n+1}\left|\Delta\left(\left(\hat{H}_{j} \circ f(z), j \in R\right)\right)\right|\right) d \theta+O(1) .
\end{aligned}
$$

By making use of Nevanlinna's lemma on logarithmic derivative generalized over $\mathbf{C}^{m}$ by A. L. Vitter [Vi77], we deduce

$$
\begin{aligned}
& \frac{1}{2 \pi \tilde{\omega}} \int_{|z|=r} \log \left(\sum_{R \subset Q,|R|=n+1}\left|\Delta\left(\left(\hat{H}_{j} \circ f(z), j \in R\right)\right)\right|\right) d \theta \\
& \quad \leqq \frac{1}{\tilde{\omega}}\left(\sum_{R \subset Q,|R|=n+1} \frac{1}{2 \pi} \int_{|z|=r} \log ^{+}\left|\Delta\left(\left(\hat{H}_{j} \circ f(z), j \in R\right)\right)\right| d \theta\right)+O(1) \\
& \quad=S_{f}(r) .
\end{aligned}
$$

From this and (3.13) the desired inequality follows. Q.E.D.

Remark on a generalization. We give a generalization of Theorem 3.1 by combining the method in $\S 2$ with that of $[\mathrm{Ng} 76]$ (cf. [Ng03] Chap. 4). Let $\pi: X \rightarrow \mathbf{C}^{m}$ be a finite analytic covering space, that is, $X$ is a normal irreducible complex space and $\pi$ is a finite mapping. Let $R$ be the ramification divisor of $\pi$, and let $p$ be the sheet number. Let $f: X \rightarrow \mathbf{P}^{n}(\mathbf{C})$ be a meromorphic mapping and take a representation $f(z)=\left[f^{0}(z), \ldots, f^{n}(z)\right]$, which is not necessarily reduced. The Wronskian $W\left(f^{0}, \ldots, f^{n}\right)$ is defined outside $R$ (cf. (3.6)) and then is extended meromorphically on $X$ (see [Fu85], [ $\mathrm{Ng} 97] \S 2(\mathrm{~b}))$.

If $f$ separates the fibers of $\pi$, we have $([\mathrm{Ng} 76])$

$$
N(r, R) \leqq(2 p-2) T_{f}(r)+O(1) .
$$

Taking account of the order of partial differentiations, one gets

$$
\left(W\left(f^{0}, \ldots, f^{n}\right)\right)+\frac{n(n+1)}{2} R \geqq 0 .
$$


As deduced (3.12) by making use of Lemmas 3.9 and 3.8, we count the divisor $\left(W\left(f^{0}, \ldots, f^{n}\right)\right)$ so that for hyperplanes $\left\{H_{j}\right\}_{j=1}^{q}$ in $N$-subgeneral position, we obtain

$$
\tilde{\omega}(q-2 N+n-1) T_{f}(r) \leqq \tilde{\omega} \sum_{j=1}^{q} N_{n}\left(r, f^{*} H_{j}\right)+\frac{n(n+1)}{2} N(r, R)+S_{f}(r) .
$$

It follows from (3.14) that

$$
\frac{n(n+1)}{2} N(r, R) \leqq n(n+1)(p-1) T_{f}(r)+O(1) .
$$

By (3.16), (3.17) and Lemma 3.3 (iii) we have

Theorem 3.18 (cf. [Ng03] Chap. 4 §3). Let $f: X \rightarrow \mathbf{P}^{n}(\mathbf{C})$ be a linearly nondegenerate meromorphic mapping. Let $\left\{H_{j}\right\}_{j=1}^{q}$ be hyperplanes of $\mathbf{P}^{n}(\mathbf{C})$ in $N$ subgeneral position. Then

$$
(q-2 N+n-1-(p-1) n(2 N-n+1)) T_{f}(r) \leqq \sum_{j=1}^{q} N_{n}\left(r, f^{*} H_{j}\right)+S_{f}(r) .
$$

\section{REFERENCES}

[DI01] R. Debalme and S. Ivashkovich, Complete hyperbolic neighborhoods in almost-complex surfaces, Internat. J. Math. 12 (2001), 211-221.

[B126] A. BLOCH, Sur les systèmes de fonctions uniformes satisfaisant à l'équation d'une variété algébrique dont l'irrégularité dépasse la dimension. J. Math. Pures Appl. 5 (1926), 19-66.

[Ca33] H. CARTAN, Sur les zéros des combinaisons linéaires de $p$ fonctions holomorphes données, Mathematica 7 (1933), 5-31.

[Ch90] W. ChEn, Defect relations for degenerate meromorphic maps, Trans. Amer. Math. Soc. 319 (1990), 499-515.

[Fu85] H. Fusimoto, Non-integrated defect relation for meromorphic maps of complete Kähler manifolds into $\mathbf{P}^{N_{1}}(\mathbf{C}) \times \cdots \times \mathbf{P}^{N_{k}}(\mathbf{C})$, Japan. J. Math. 11 (1985), 233-264.

[Fu93] H. Fusmoto, Value Distribution Theory of the Gauss Map of Minimal Surfaces in $\mathbf{R}^{m}$, Aspects of Math. E21, 1993.

[Ko98] S. Kobayashi, Hyperbolic Complex Spaces, Grund. der Math. Wissen. 318, SpringerVerlag, Berlin, 1998.

[Ko01] S. Kobayashi, Almost complex manifolds and hyperbolicity, Dedicated to Shiing-Shen Chern on his 90th birthday, Results Math. 40 (2001), 246-256.

[Ko03] S. Kobayashi, Natural connections in almost complex manifolds, Explorations in complex and Riemannian geometry, Contemp. Math. 332 (2003), Amer. Math. Soc., Providence, RI, 153-169.

[Ko04] S. Kobayashi, Problems related to hyperbolicity of almost complex structures, to appear in Proc. OKA 100 Conference Kyoto/Nara 2001, Complex Analysis in Several Variables, Adv. Studies in Pure Math. Math. Soc. Japan, Tokyo, 2004.

[Nc82a] I. E. NochKA, Defect relations for meromorphic curves (in Russian), Izv. Akad. Nauk Moldav. SSR Ser. Fiz.-Tekhn. Mat. Nauk (1982) no. 1, 41-47. 
[Nc82b] E. I. NochKA, On a theorem from linear algebra (in Russian), Izv. Akad. Nauk Moldav. SSR Ser. Fiz.-Tekhn. Mat. Nauk (1982) no. 3, 29-33.

[Nc83] E. I. NochKa, On the theory of meromorphic functions, Sov. Math. Dokl. 27 (1983), 377381 .

[Ng76] J. Noguchi, Meromorphic mappings of a covering space over $\mathbf{C}^{m}$ into a projective variety and defect relations, Hiroshima Math. J. 6 (1976), 265-280.

[Ng97] J. NoguchI, Nevanlinna-Cartan theory over function fields and a Diophantine equation, J. reine angew. Math. 487 (1997), 61-83; Correction to the paper, Nevanlinna-Cartan theory over function fields and a Diophantine equation, J. reine angew. Math. 497 (1998), 235.

[Ng03] J. NoguchI, Nevanlinna Theory in Several Variables and Diophantine Approximation (in Japanese), Kyoritu Publ. Co., Tokyo, 2003.

$\left[\mathrm{NO} \frac{84}{90}\right]$ J. Noguchi AND T. OchiaI, Geometric Function Theory in Several Complex Variables, Japanese edition, Iwanami, Tokyo, 1984; English Translation, Transl. Math. Mono. 80, Amer. Math. Soc., Providence, Rhode Island, 1990.

[NWY02] J. Noguchi, J. Winkelmann AND K. YamanoI, The second main theorem for holomorphic curves into semi-Abelian varieties, Acta Math. 188 (2002), 129-161.

[Oc77] T. OCHIAI, On holomorphic curves in algebraic varieties with ample irregularity, Invent. Math. 43 (1977), 83-96.

[Vi77] A. L. VitTER, The lemma of the logarithmic derivative in several complex variables, Duke Math. J. 44 (1977), 89-104.

Graduate School of Mathematical Sciences

THE UNIVERSITY OF TOKYO

Komaba, Meguro, ToKyo 153-8914

e-mail: noguchi@ms.u-tokyo.ac.jp 